Please do not remove this page

RMIT

UNIVERSITY

\title{
A reliability study of the new back strain monitor based on clinical trials
}

Ronchi, Andrew; Lech, Margaret; Taylor, Nick; Cosic, Irena

https://researchrepository.rmit.edu.au/esploro/outputs/9921863834801341/filesAndLinks?institution=61RMIT_INST\&index=null

Ronchi, A., Lech, M., Taylor, N., \& Cosic, I. (2008). A reliability study of the new back strain monitor based on clinical trials. 30th Annual International Conference of the IEEE Engineering in Medicine and Biology Society, 693-696. https://doi.org/10.1109/IEMBS.2008.4649247

Published Version: https://doi.org/10.1109/IEMBS.2008.4649247

Repository homepage: https://researchrepository.rmit.edu.au

(c) 2008 IEEE. Personal use of this material is permitted. However, permission to reprint/republish this material for advertising or promotional purposes or for creating new collective works for resale or redistribution to servers or lists, or to reuse any copyrighted component of this work in other works must be obtained from the IEEE.

Downloaded On 2023/04/26 23:42:43 +1000 


\title{
A Reliability Study of the New Back Strain Monitor Based on Clinical Trials.
}

\author{
A. J. Ronchi, M. Lech, N. F. Taylor and I. Cosic, Senior Member, IEEE
}

\begin{abstract}
A new Back Strain Monitor (BSM) device has been developed in order to measure, record and analyze movements of the lower back. The purpose of this study was to examine the inter-tester and the intratester reliability of the movement measurements given by the BSM accelerometers, and compare it with the reliability of two other conventional measurement methods: the Double Inclinometer method (DI) and the Modified-Modified Schober (MMS) method. The clinical studies included 23 participants (16 males, 7 females) with no recent history of lower back pain, who wore the device during a combination of different anatomical movements (flexion, extension, left lateral flexion and right lateral flexion of the lumber spine). The tests were conducted by three therapists (testers). The reliability results for the BSM accelerometers clearly outperform the results obtained for the DI and the MMS methods. The inter-tester reliability gives the Intra-Class Correlation (ICC) value of 0.95 for the BSM flexion, 0.89 for the DI flexion and 0.74 for the MMS. The intra-tester reliability gives the ICC value of 0.99 for BSM flexion, 0.94 for DI flexion and 0.77 for the MMS. The BSM accelerometers were highly reliable in assessing back movements, measuring these movements with less error than the DI and MMS methods.
\end{abstract}

\section{INTRODUCTION}

Lower back pain continues to be a major problem with studies stating that $20-25 \%$ of all injuries affect the lower back and that these lower back injuries account for $40 \%$ of compensation costs [24]. Once injured the recurrence rate of lumbar spine injuries is $60-85 \%$ within the first three years [25]. Previous techniques have attempted to quantify the movements of the lower back in real time and with minimal impedance to the wearer. C.Snijders [2] looked at continuous

Manuscript received April 7, 2008

A. J. Ronchi is with the School of Electrical and Computer Engineering, RMIT University, GPO Box 2476V Melbourne Victoria 3001 Australia (email: s3029015@student.rmit.edu.au)

M. Lech is with the School of Electrical and Computer Engineering, RMIT University, GPO Box 2476V Melbourne Victoria 3001 Australia (corresponding author: tel. 61-3-9925-1028, fax 61-3-9925-2007, margaret.lech@rmit.edu.au)

N. F. Taylor is with the Musculoskeletal Research Centre, La Trobe University, Bundoora Victoria 3086, AUSTRALIA (e-mail: n.taylor@latrobe.edu.au)

I. Cosic is with the School of Electrical and Computer Engineering, RMIT University, GPO Box 2476V Melbourne Victoria 3001, Australia (email: irena.cosic@ rmit.edu.au). measurements of spine movements and attached various sensors to the spine to measure movements over time. W.Marras [22] built the Lumbar Motion Monitor and used this device to analyze over 400 jobs in order to assess the three dimensional trunk motion's role in the development of lower back dysfunction.

The Back Strain Monitor (BSM) is a new device for measuring lumbar spine movement, within a controlled setting. The device is unobtrusive, compact, easy to apply and is designed to measure movement in three planes (Flexion, Lateral Flexion and Rotation), muscle activity around the lumbar spine and vibration affecting the lumbosacral region. The aim of this study is to assess the reliability of the BSM for measuring flexion/extension and left/right lateral flexion of the lumbar spine.

A reliable dynamic recording of lumbar spine movement in a real-life occupational environment represents a complex and challenging task. An appropriate measuring protocol for realtime lumbar spine movement has to be capable of a quantitative registration and measurement of factors including the spine's three dimensional movement, body weight, gravity, amount of active and passive support of the spine, weight being lifted, various environmental factors, as well as the psychological and social aspects of the working environment.

A number of different existing methods for measuring lower back movement were reviewed in order to find techniques to compare the BSM with. The Byplanar Radiograghy due to radiation concerns and lack of portability, was not appropriate. The Flexirule uses a flexible ruler placed on the spine to follow the curvature of the lumbar spine. There seems to be trouble with obtaining accurate tangents with this method and it was shown to have no inter tester reliability [23]. The finger to floor method is quick and simple and shown to have inter-tester reliability $[6,7,12]$, but was deemed not valid by Moll and Wright in [8], and it is not comparable from one subject to another. Two methods were finally chosen for comparison with the BSM: the ModifiedModified Schober (MMS) method and the Double Inclinometer (DI) method. Both methods have been shown to have inter and intra-tester reliability [7,9,10]. The ModifiedModified Schober method uses a flexible tape measure and measures the change in skin stretch when the lumbar spine flexes from a neutral position to a fully flexed position. The landmarks are at the lumbosacral junction and a line drawn $150 \mathrm{~mm}$ superior to this point. The Double Inclinometer 
technique measures the angular difference between the upper lumbar spine and the lower lumbar spine in a given position and subtracts one from the other. These measurements are then retaken once a movement has been performed and again subtracted from one another. The difference in degrees from the initial measurement to the subsequent measurement represents the amount of lumbar spine movement.

Most of the currently available lumbar movement measuring devices have the disadvantage of being large, cumbersome, expensive and lack the capability to analyse the measurement data in real time. Such devices cannot be easily used over long periods of time in real occupational environments.

The new BSM eliminates most of these drawbacks, however, the reliability and validity of the BSM needed to be reviewed to establish whether it can be used as a diagnostic/ therapeutic tool.

The paper analyses lumbar spine movement and compares the measurement results given by two pre-existing and well recognized methods: MMS and DI with the results given by the new BSM. The reliability tests were performed using a clinical trial protocol which has already been field tested. The trials were conducted within a controlled setting.

The comparison between the BSM measurements and the MMS and DI measurements was determined using the intraand inter-tester reliability measure based on the intraclass correlation coefficient (ICC) [21].

\section{METHODS}

\section{A. The Back Strain Monitor}

The Back Strain Monitor is a recently developed electronic and programmable device. The BSM can be used by patients presenting with low back pain or patients at risk of developing low back pain, to monitor movement, estimate the amount of strain on the lower back, and to provide realtime feedback to the patient about potentially provocative postures for their lumbar spine. Real-time feedback allows the patient to immediately correct the body posture or to stop the physical activity that may carry a higher risk of injury.

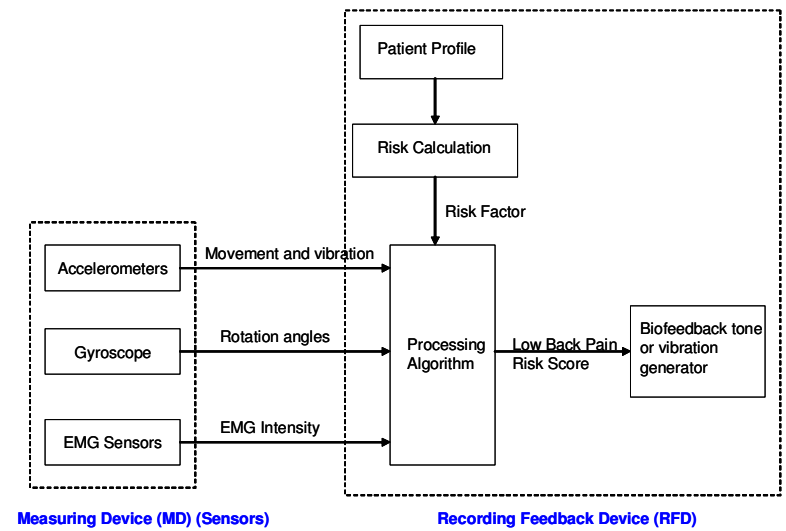

Picture1. Functional Flowchart of the BSM.
As illustrated in Picture 1, the BSM consists of two parts: the Measuring Device (MD) and the Recording Feedback Device (RFD). The Measuring Device contains the transducer components collecting raw, real-time movement and muscle activity data from the patients via sensors placed on the patient's body. The Recording Feedback Device is an electronic, programmable component that uses a numerical algorithm to extract and process information from the sensors, and from the Patient Profile stored in the memory, to calculate a low back pain risk score. When the risk score exceeds a certain threshold value, a real-time feedback signal (sound or vibration) is activated to inform the patient of the excessive load or high risk activity their low back may be experiencing.

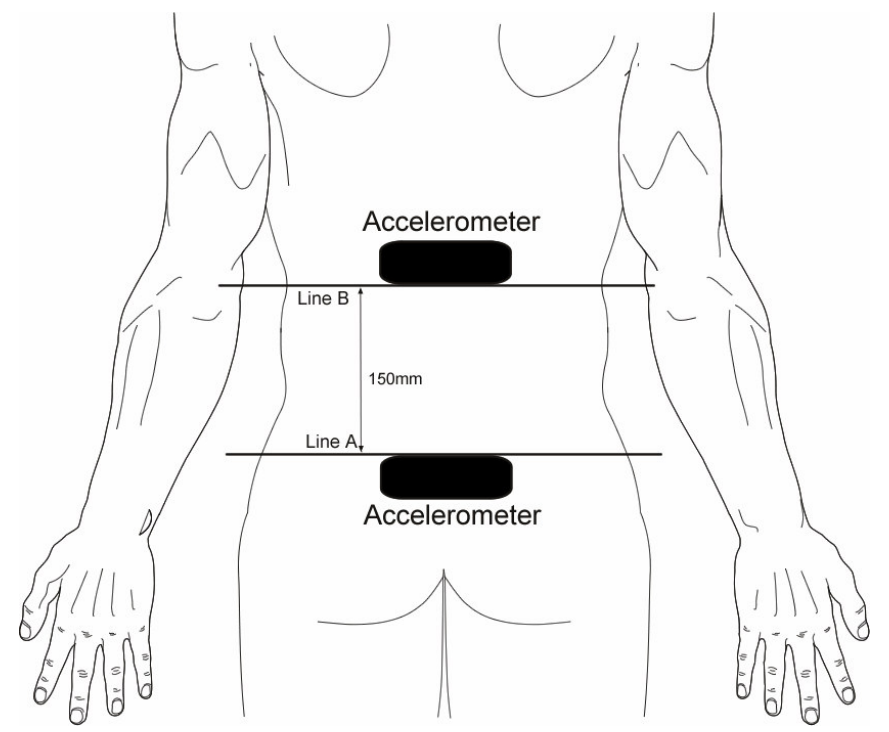

Picture2. The BSM fitted on the participant's back.

\section{B. The reliability testing in a controlled setting}

The aim of the experiment was to assess the inter- and intratester reliability of the BSM, MMS and DI methods.

\section{Participants}

The sample study included participants who never suffered from the Lower back Pain (LBP) as well as participants who suffered from LBP in the past. Those who suffered from LBP in the past were accepted only under the condition that the pain had not occurred for at least 3 months and it was not occurring during the trial. All participants were examined by a physiotherapist immediately before the trial to ensure that each subject had full pain free movement during their warm up period prior to the trial. The participants were also required to have no history of spinal surgery to the lumbar, thoracic or cervical spine. A sample of 23 participants including 16 males (average age of 40) and 7 females (average age of 42) were tested. The participant's age ranged from 21 to 62 years (average age 40.4). The participants were recruited on a voluntary basis. No payment was offered. The participants were spread across different occupational groups. Prior to the tests, the participants were briefly interviewed by a practitioner to confirm that they 
fulfilled the inclusion criteria. All participants signed a consent form for the project which had received institutional ethics approval.

\section{Testers}

The study was carried out within a physiotherapy centre. During the tests, the participants were assisted by three experienced practitioners with 12 to 17 years of clinical experience. The practitioners fitted the BSM device and instructed movements via the study protocol. They also conducted the DI and MMS measurements. A scribe was provided to improve efficiency and to allow the tester's full focus on the subject and their explanation of the movements required. Each tester had their own room with no visual contact with the other testers or subjects to ensure the study was double blind. The subjects were allocated a tester in a random order and the landmarks were removed completely prior to the subject moving from one tester to another.

\section{Fitting the BSM device}

The BSM devices were installed on the participant's back such that the accelerometers were placed above line $\mathrm{A}$ and below line B (see Picture 2).

\section{Experimental procedure}

The experimental procedure included the following steps:

\section{Subject Starting Position}

Each subject before commencing movements, was asked to ensure the following: stand facing the door of the room with head and shoulders straight; feet shoulder width apart; arms relaxed by side; and legs and trunk in an erect but relaxed stance.

\section{Movements}

The following four movements were performed in a random sequence to avoid any potential bias.

\subsection{Lumbar spine Flexion}

\subsection{Lumbar spine extension:}

\subsection{Lumbar spine Lateral Flexion:}

\subsection{Thoraco-lumbar rotation:}

\section{RESULTS AND DISCUSSION}

The statistical analysis reviewed measurements in two ways. Firstly, the Inter-tester Reliability, as described by D.Streiner [1], was used to determine the variability between different testers. Secondly, the Intra tester Reliability [1], was used to determine the variability between the same tester but on different days.

In both cases, methods described by Shrout and Fleiss [21] were used to derive the intraclass correlation coefficient (ICC) for each aspect of movement: flexion, extension, left lateral flexion and right lateral flexion.

\section{A. The inter-tester reliability results}

The inter-tester reliability results for the flexion movement are summarized in Table I along with the results for extension of the lumbar spine and lateral flexion of the lumbar spine. Note that there are no results for the MMS for extension and lateral flexion's as this technique is not well recognized as a method for measuring extension and lateral flexion.

Table I. Results of the inter-tester reliability test. The ICC values for the BSM accelerometers, DI and MMS.

\begin{tabular}{|l|c|c|c|}
\hline Type of movement & ICCs for BSM & ICCs for DI & ICCs for MMS \\
\hline Flexion Plane & 0.954 & 0.892 & 0.736 \\
\hline Extension Plane & 0.947 & 0.909 & N/A \\
\hline Left Lateral Flexion & 0.887 & 0.845 & N/A \\
\hline Right Lateral Flexion & 0.859 & 0.834 & N/A \\
\hline
\end{tabular}

The results in Table I indicate that the BSM accelerometers showed the best overall reliability for all four types of the lower back movement. The ICC values for the BSM accelerometers ranged from 0.859 to 0.954 .

The DI method shows lower overall reliability compared to the BSM, and the method with the poorest results was the MMS, with the ICC value of 0.736 .

\section{B. The intra-tester reliability results}

The intra-class correlation was again used to compare the results obtained by the same tester but on different days. One of the three testers repeated the BSM accelerometer method, the DI and the MMS method measurements on twenty two of the twenty three subjects, five weeks after the first testing, in order to reduce memory effect.

Table II. Results of the intra-tester reliability test. The ICC values for the BSM accelerometers, DI and MMS.

\begin{tabular}{|l|l|}
\hline \multicolumn{1}{|c|}{ Method } & ICC \\
\hline BSM_extension & 0.98191 \\
\hline BSM_flexion & 0.99315 \\
\hline BSM_llflexion & 0.89168 \\
\hline BSM_rlflexion & 0.93040 \\
\hline DI_extension & 0.95345 \\
\hline DI_flexion & 0.94487 \\
\hline DI_llflexion & 0.88403 \\
\hline DI_rlflexion & 0.86140 \\
\hline MMS_flexion & 0.77018 \\
\hline
\end{tabular}

The same settings and protocol were used; however the order of subjects was randomized again to avoid any memory effect. The intra-tester reliability results are summarized in Table II. 


\section{CONCLUSION}

A new Back Strain Monitor (BSM) device has been developed in order to measure, record and analyze movements of the lower back within a real job setting, with minimal inconvenience to the wearer.

The purpose of this study was to examine the inter-tester and the intra-tester reliability of the movement measurements given by the BSM accelerometers. The reliability tests of the BSM accelerometers were compared with the reliability of two other conventional measurement methods: the Double Inclinometer method (DI) and the Modified-Modified Schober (MMS) method.

The clinical studies included 23 participants (16 males, 7 females) with no recent history of lower back pain. During the tests the participants wore the device during a combination of different anatomical movements (flexion, extension, left lateral flexion and right lateral flexion of the lumber spine). The tests were conducted by three experienced practitioners (testers).

The reliability of the tested methods was measured using the Intra-Class Correlation Coefficient (ICC).

The inter-tester reliability for the BSM ranges from 0.859 to 0.954 .

The inter-tester reliability for the DI ranges from 0.834 to 0.909 .

For the MMS it was only possible to obtain one value of the inter-tester ICC (flexion plane) of 0.736.

The intra-tester reliability for the BSM ranges from 0.89 to 0.99 .

The intra-tester reliability for the DI ranges from 0.86 to 0.95 .

Again, for the MMS it was only possible to obtain one value of the inter-tester ICC (flexion plane) of 0.77 .

In conclusion, the reliability results for the BSM accelerometers were positive, showing slightly higher reliability than the DI method and moderately higher reliability than the MMS method. The results indicate that the BSM can reliably measure movement of the lower back. Further validity testing is required before the BSM can be considered as a useful management tool for back pain sufferers.

\section{REFERENCES}

[1] D.Streiner, Health Measurement Scales, 3rd Ed, 2003

[2] Snijders, C. J., M. Van Riel, et al. (1987). "Continuous measurements of spine movements in normal working situations over periods of 8 hours or more." Ergonomics 30(4): 639-653.
[3] Mitton, D., C. Landry, et al. (2000). "3D reconstruction method from biplanar radiography using non-stereocorresponding points and elastic deformable meshes." Medical and Biological Engineering and Computing 38(2): 133-139.

[4] Tibrewal, S. B., M. J. Pearcy, et al. (1985). "A prospective study of lumbar spinal movements before and after discectomy using biplanar radiography. Correlation of clinical and radiographic findings." Spine(Philadelphia, PA. 1976) 10(5): 455-460.

[5] Stokes, I. A. and J. W. Frymoyer (1987). "Segmental motion and instability." Spine 12(7): 688-91.

[6] Frost, M., S. Stuckey, et al. (1982). "Reliability of measuring trunk motions in centimeters." Phys Ther 62(10): 1431-7.

[7] Newton, M. and G. Waddell (1991). "Reliability and validity of clinical measurement of the lumbar spine in patients with chronic low back pain." Physiotherapy 77(12): 796-800.

[8] Moll, J. (1976). Measurement of spinal movement, The lumbar spine and back pain. London, Sector Publishing.

[9] Million, R., K. H. Nilsen, et al. (1981). "Evaluation of low back pain and assessment of lumbar corsets with and without back supports." British Medical Journal 40(5): 449.

[10] Biering-Sorenson, F. (1984). "Physical measurement as a risk factor for back trouble over a one year period." Spine 9: 106-109.

[11] Merritt, J. L., T. J. McLean, et al. (1986). "Measurement of trunk flexibility in normal subjects: reproducibility of three clinical methods." Mayo Clinic proceedings 61(3): 192-197.

[12] Gill, K., M. H. Krag, et al. (1988). "Repeatability of four clinical methods for assessment of lumbar spinal motion." Spine 13(1): 50-3.

[13] Rae, P. (1984). "A simple technique for measuring lumbar spinal flexion." J R Coll Surg Edinb 29(5): 281-284.

[14] Keeley, J., T. G. Mayer, et al. (1986). "Quantification of lumbar function. V: Reliability of range-of-motion measures in the sagittal plane and an in vivo torso rotation measurement technique." Spine(Philadelphia, PA. 1976) 11(1): 31-35.

[15] Beattie, P., J. M. Rothstein, et al. (1987). "Reliability of the attraction method for measuring lumbar spine backward bending." Phys Ther 67(3): 364-9.

[16] Gauvin, M. G., D. L. Riddle, et al. (1990). "Reliability of clinical measurements of forward bending using the modified fingertip-tofloor method." Physical Therapy 70(7): 443.

[17] Paquet, N., F. Malouin, et al. (1991). "Validity and reliability of a new electrogoniometer for the measurement of sagittal dorsolumbar movements." Spine 16(5): 516-9.

[18] Portek I, P. M. (1983). "Correlation between radiographic and clinical measurement of lumbar spine movement." British Journal of Rheumatology 22(4): 197-205.

[19] Mayer, T. G., A. F. Tencer, et al. (1984). "Use of noninvasive techniques for quantification of spinal range-of-motion in normal subjects and chronic low-back dysfunction patients." Spine 9(6): 58895.

[20] Keating, J. and T. Matyas (1998). "Unreliable inferences from reliable measurements." Aust J Physiother 44(1): 5-10.

[21] Shrout, P. E. and J. L. Fleiss (1979). "Intraclass correlations: uses in assessing rater reliability." Psychol Bull 86(2): 420-8.

[22] Marras W, (1999), The role of dynamic three-dimensional trunk motion in occupationally-related low back disorders, Spine Vol 18 , No.5, 617-628, 1993.

[23] Lovell D., Frankin W.; Personius, Walter J.; Rothstein, Jules M.n (1989) Reliability of clinical measurements of lumbar lordosis taken with a flexible rule. Physical Therapy, 1989.

[24] Marras, W. S., S. A. Lavender, et al. (1995). "Biomechanical risk factors for occupationally related low back disorders." Ergonomics 38(2): 377-410.

[25] Tan, J. C., M. Parnianpour, et al. (1993). "Isometric maximal and submaximal trunk extension at different flexed positions in standing. Triaxial torque output and EMG." Spine 18(16): 2480-90. 